\title{
Vagal innervation of the gastrointestinal tract arises from dorsal motor nucleus while that of the heart largely from nucleus ambiguus in the cat
}

\author{
J.H. Hsieh ${ }^{\mathrm{a}, \mathrm{b}}$, R.F. Chen ${ }^{\mathrm{c}}$, J.J. Wu ${ }^{\mathrm{b}}$, C.T. Yen ${ }^{\mathrm{c}}$, C.Y. Chai ${ }^{\mathrm{a}, *}$ \\ ${ }^{a}$ Institute of Biomedical Sciences, Academia Sinica, Taipei 11529, Taiwan \\ ${ }^{\mathrm{b}}$ Institute of Biomedical Engineering, Chung Yuan Christian University, Chung Li, Taiwan \\ ${ }^{\mathrm{c}}$ Department of Zoology, National Taiwan University, Taipei, Taiwan
}

Received 3 February 1997; revised 16 January 1998; accepted 21 January 1998

\begin{abstract}
The origin of medullary cells that form the cardiac vagal branch and the vagal branches in the lower thorax innervating the gastrointestinal (GI) tract was studied using horseradish peroxidase (HRP), a retrograde transport tracer in the cat. The distributions of parasympathetic postganglionic neurons of the heart were studied with acetylcholinesterase histochemistry. Intracardiac ganglionic neurons were found mainly in the connective tissue surrounding the base of the pulmonary arteries and in an area in and dorsal to the interatrial septum. Following injection of HRP into the subepicardum where most of the cardiac postganglionic neurons reside, $91 \%$ of the labelled neurons were found bilaterally distributed in the nucleus ambiguus (NA). A small population of labelled neurons was found in the dorsal motor nucleus of the vagus (DMV) and an intermediate zone (IZ) between the two nuclei. When HRP was injected into the left or right cardiopulmonary vagus branch, labelled neurons were found exclusively in the ipsilateral NA, DMV and IZ with a predominance in the NA. In the thorax, after they course around the heart, the left and right thoracic vagus nerves divides into a left and a right branch, respectively. The left branch of the left thoracic vagus joins the left branch of the right thoracic vagus to form the anterior vagus nerve at $3 \mathrm{~cm}$ above the diaphragm. The right branch of the right thoracic vagus nerve joins the right branch of the left thoracic vagus to form the posterior vagus nerve. After application of HRP into the right or the left branch of the left thoracic vagus, HRP labelled cells were found in the left DMV. Similarly, after application of HRP into the left or the right branch of the right thoracic vagus, labelled cells were found in the right DMV. On the other hand, when HRP was injected into the anterior vagus, labelled neurons were found bilaterally in the DMV. This suggests that all rostral branches of the thoracic vagus have their origin in the ipsilateral DMV, and intermixing occurs only at the caudal level near the diaphragm. Findings of the present experiments suggest that parasympathetic preganglionic neurons innervating the GI tract are located exclusively in the DMV while those of the heart are located mainly in the NA. Within the DMV, GI vagal neurons were found medially from the level 0-2.5 $\mathrm{mm}$ rostral to the obex. In contrast, cardiac vagal neurons were found in the lateral edge of the DMV at the level 0-1 mm rostral to the obex. (C) 1998 Elsevier Science B.V. All rights reserved.
\end{abstract}

Keywords: Parasympathetic preganglionic neuron; Horseradish peroxidase; Retrograde tracing; Intracardiac ganglionic neuron

\section{Introduction}

The vagus nerve extensively innervates the thoracic and abdominal viscera. As early as 1845 , the Weber brothers began to study the influences of vagus on the heart (Hoff, 1936). Subsequently, the vagus nerve has been found to exert an important control over the cardiac rhythm, rate, and contractility of the heart. The vagal cardiac innervation of vagus, however, has long been a source of controversy. Uncertainties remain regarding the origin and distribution

\footnotetext{
* Corresponding author. Tel.: +886 2 27899105; fax: +886 2 27829224.
}

of its preganglionic motoneurons within the medulla oblongata that innervate specific cardiac targets (Hopkins and Ellenberger, 1994).

Projections from the nucleus ambiguus (NA) and the dorsal motor nucleus of the vagus (DMV) effecting parasympathetic influences over cardiac functions have been extensively studied. Various approaches have been used, including, physiological (Geis and Wurster, 1980; Norman et al., 1985), tract tracing (Ciriello and Calaresu, 1982; Getz and Sirnes, 1949; Norman et al., 1985), and degeneration techniques (Massari et al., 1995). Location of cardiac vagal preganglionic neurons have been found in both DMV and NA (Cabot et al., 1991; Ciriello and 
Calaresu, 1982; Getz and Sirnes, 1949; Geis and Wurster, 1980; Izzo et al., 1993; Massari et al., 1995; Nosaka et al., 1982; Standish et al., 1994). Some investigators consider that preganglionic neurons are located primarily in NA (McAllen and Spyer, 1976; Stuesse, 1982, 1984) while others have suggested that they lie primarily in DMV (Cohen et al., 1970; Todo et al., 1977). Species variation may account for some of the discrepancies. For example, in the pigeon, vagal neurons exist exclusively in the DMV (Cohen et al., 1970). There may be a dominant contribution from NA in the cat, dog and pig (Hopkins, 1987); whereas both NA and DMV may be important in the rat (Izzo et al., 1993; Nosaka et al., 1982; Standish et al., 1994) and rabbit (Jordan et al., 1982).

Another possible variation may arise from differences in injection sites. Injection of horseradish peroxidase (HRP), a retrograde tracer, into the cardiopulmonary branches of the vagus usually produces labelling of neurons in both DMV and NA (Ciriello and Calaresu, 1982; Maqbool et al., 1991). Selective injection into the heart of the cat, however, has produced labelling predominantly in NA (Stuesse, 1982). Therefore, the first objective of the present study was to compare the pattern of HRP labelled neuron resulting from selective injection of the tracer into either the postganglionic neuronal pool of the heart or the cardiopulmonary branch of the vagus in cats.
The role of the vagus nerve in gastrointestinal (GI) function has also attracted considerable attention, but comparatively less effort has been placed on this subject (Pagani et al., 1985). Anatomical studies have shown that the NA contains cell bodies with their preganglionic vagal fibers projecting to GI tract (Gwyn et al., 1985; Jou et al., 1993; Kalia and Mesulam, 1980; Takayama et al., 1982). Williford et al. (1981) reported that microinjections of bicuculline, a gamma aminobutyric acid (GABA) antagonist, into the NA of cats produces a significant increase in the motility of antrum and pyloric sphincter. These effects were reversed by microinjections of muscimol, a GABA agonist, into the same brain area. Thus, the effect of bicuculline was interpreted to be a blockade of the inhibitory GABAergic tone on the NA, thus resulting in an augmentation of parasympathetic activity in the stomach.

On the other hand, neuroanatomical studies have shown that DMV contains one of the most important cell groups innervating the stomach (Bieger and Hopkins, 1987; Laughton and Powley, 1987; Pagani et al., 1988; Takayama et al., 1982; Yamamoto et al., 1977), pancreas (Pagani et al., 1984; Rinaman and Miselis, 1987) and other abdominal organs (Kalia and Mesulam, 1980; Stuesse, 1982). Using HRP, Fox and Powley (1985) clearly demonstrated a longitudinal columnar organization within DMV representing separate vagal innervation of the abdominal organs

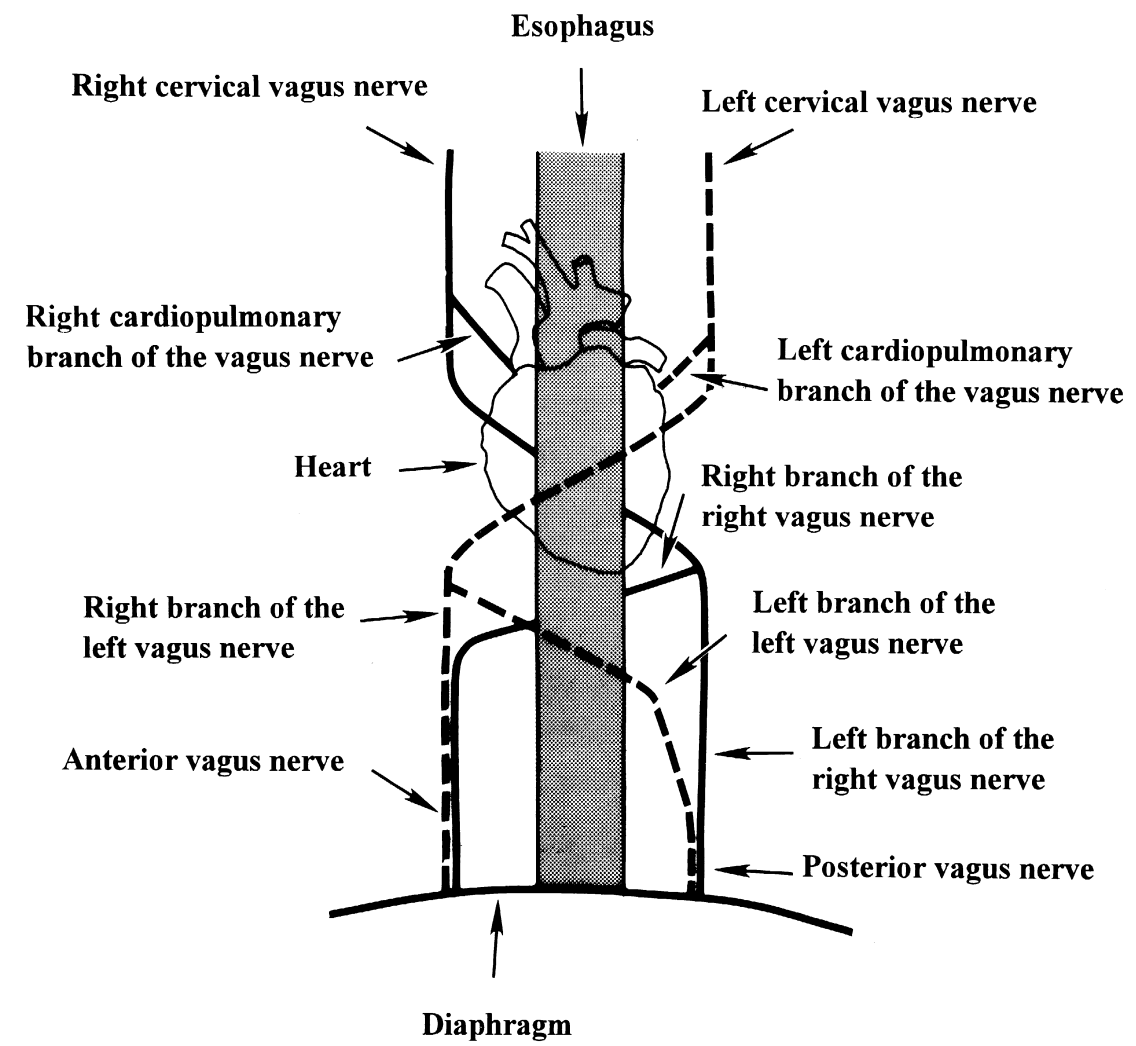

Fig. 1. The diagrammatic representation of vagus branches injected with HRP in the present study. These included the left cervical vagus, the right and the left cardiopulmonary branches of the vagus, the right and the left branches of the right thoracic vagus, the right and the left branches of the left thoracic vagus, and the anterior vagus nerves. 
in a topographical manner. Okumura and Namiki (1990) reported that in rats the parasympathetic preganglionic neurons innervating the stomach are site-specifically organized within the DMV.

Based on the aforementioned considerations, the second objective of the present study was to re-examine the origin of the vagus nerve to the GI tract (Dennison et al., 1981; Ewart et al., 1988; Getz and Sirnes, 1949; Izzo et al., 1993; Kalia and Mesulam, 1980; Kalia and Sullivan, 1982; Karim et al., 1984; Takayama et al., 1982; Yamamoto et al., 1977). Unlike most of the studies of examining vagal innervation in the abdomen, in which the tracer was injected either into a branch of the abdominal vagus or into the target organs (Ciriello and Calaresu, 1982), we studied the origin of the vagal branches to the GI tracts by injecting HRP into five different vagal branches within the lower thorax. In this way, we sought to determine if the GI tract and the heart were differentially innervated by the
DMV and the NA. Furthermore, new knowledge regarding the branching pattern of the vagus in the lower thoracic level and the location of the cell bodies of these branches was also explored.

\section{Materials and methods}

\subsection{Distribution of the intracardiac ganglionic neurons (acetylcholinesterase labelled) in the heart}

The purpose of this experiment was to pin point the location of the densest vagal postganglionic neurons in the heart. Three cats were anesthetized intraperitoneally with sodium pentobarbital $(35 \mathrm{mg} / \mathrm{kg})$. Each cat was perfused through the descending aorta with $0.9 \%$ physiological saline, followed by 21 fixative of $1 \%$ paraformaldehyde and $2.5 \%$ glutaraldehyde in $0.1 \mathrm{M}$ phosphate buffer $(\mathrm{pH}$
A

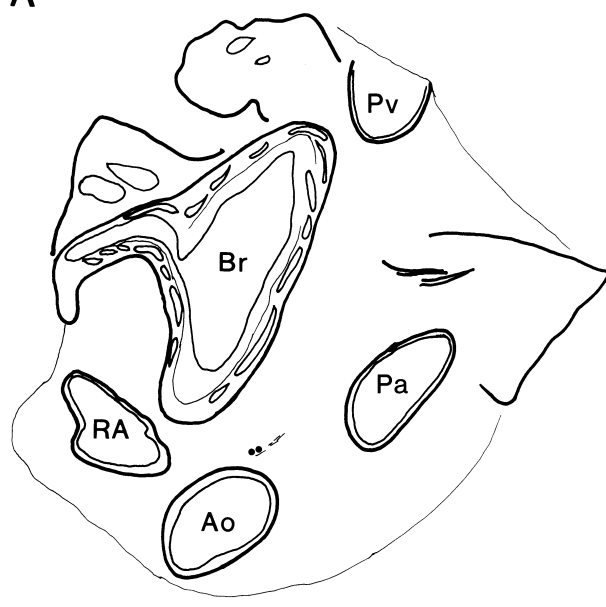

C

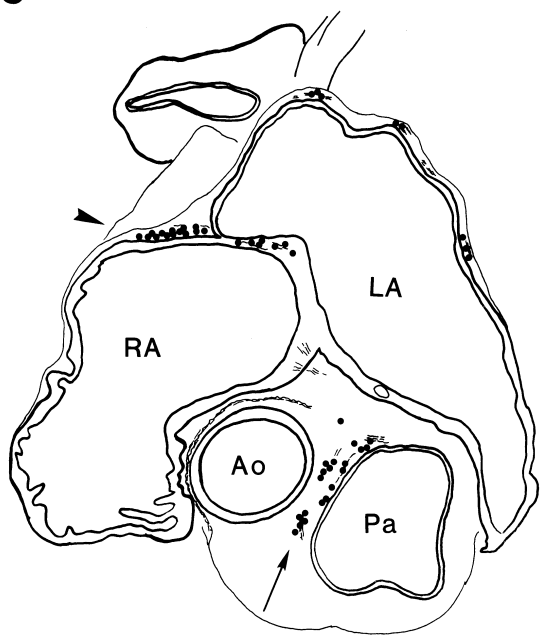

B

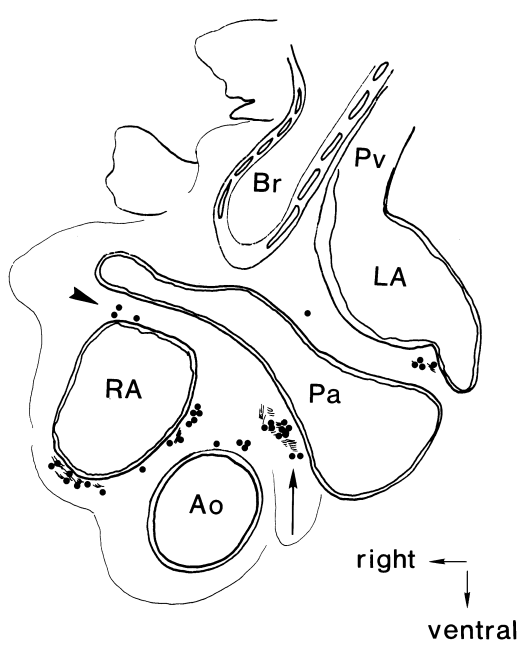

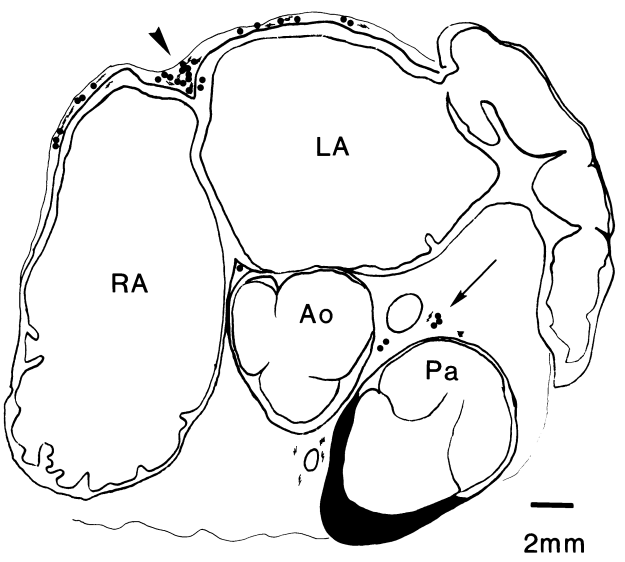

Fig. 2. Distribution of the intracardiac ganglionic neurons in the heart labelled with acetylcholinesterase. A-D show various levels of the heart in transverse sections from its base (A) to the apex (D). Two groups of the postganglionic neurons were found. A ventral group (arrows) located between the aorta (Ao) and pulmonary artery ( $\mathrm{Pa}$ ) shown in $\mathrm{A}, \mathrm{B}, \mathrm{C}$ and $\mathrm{D}$. The other group (arrowheads) located dorsal to the atria and within the intra-atrial septum as shown in $\mathrm{B}, \mathrm{C}$ and $\mathrm{D}$. In D the region marked in black is the right ventricular wall. Br, Bronchus; LA, left atrium; Pv, pulmonary vein. 
7.3). The heart (myocardium), large vessels (aorta, pulmonary artery, pulmonary veins and vena cava), inferior trachea and a portion of lung and esophagus in the region near the heart were removed and stored 1-2 days in the same fixative with $30 \%$ sucrose at $4{ }^{\circ} \mathrm{C}$. The tissues were embedded in gelatin and stored 3-5 days in phosphate buffer with $30 \%$ sucrose. Serial frozen sections $(100 \mu \mathrm{m})$ were cut transversely and processed for histochemical demonstration of the acetylcholinesterase reaction (Woolf and Butcher, 1981). An alternate series of sections were mounted on gelatin-subbed slides and allowed to air dry. The sections were cleared with xylene and coverslipped. The serial sections were examined systematically and the distribution of acetylcholinesterase labelled neurons in the heart was plotted onto outlines of sections using camera lucida attached to an stereomicroscope (Wild M3Z).

\subsection{Identification of the origin of various thoracic vagal} branches by retrograde HRP technique

A total of 38 cats were used. Each animal was anesthetized intraperitoneally with pentobarbital $(35 \mathrm{mg} / \mathrm{kg})$, intubated with a endotracheal tube (Mallinckrodt, size $4.5-5.0 \mathrm{~mm}$ i.d.) and artificially ventilated.

\subsubsection{Injection of HRP into the subepicardium}

After identification of the locations of cardiac ganglia, intracardiac injections of HRP were administered at these locations to trace the origin of the cardiac vagal preganglionic neurons. Six cats were thoracotomized between the fourth and fifth ribs. In each animal after the pericardium was incised by means of a $10-\mu$ l Hamilton syringe, $20 \%$

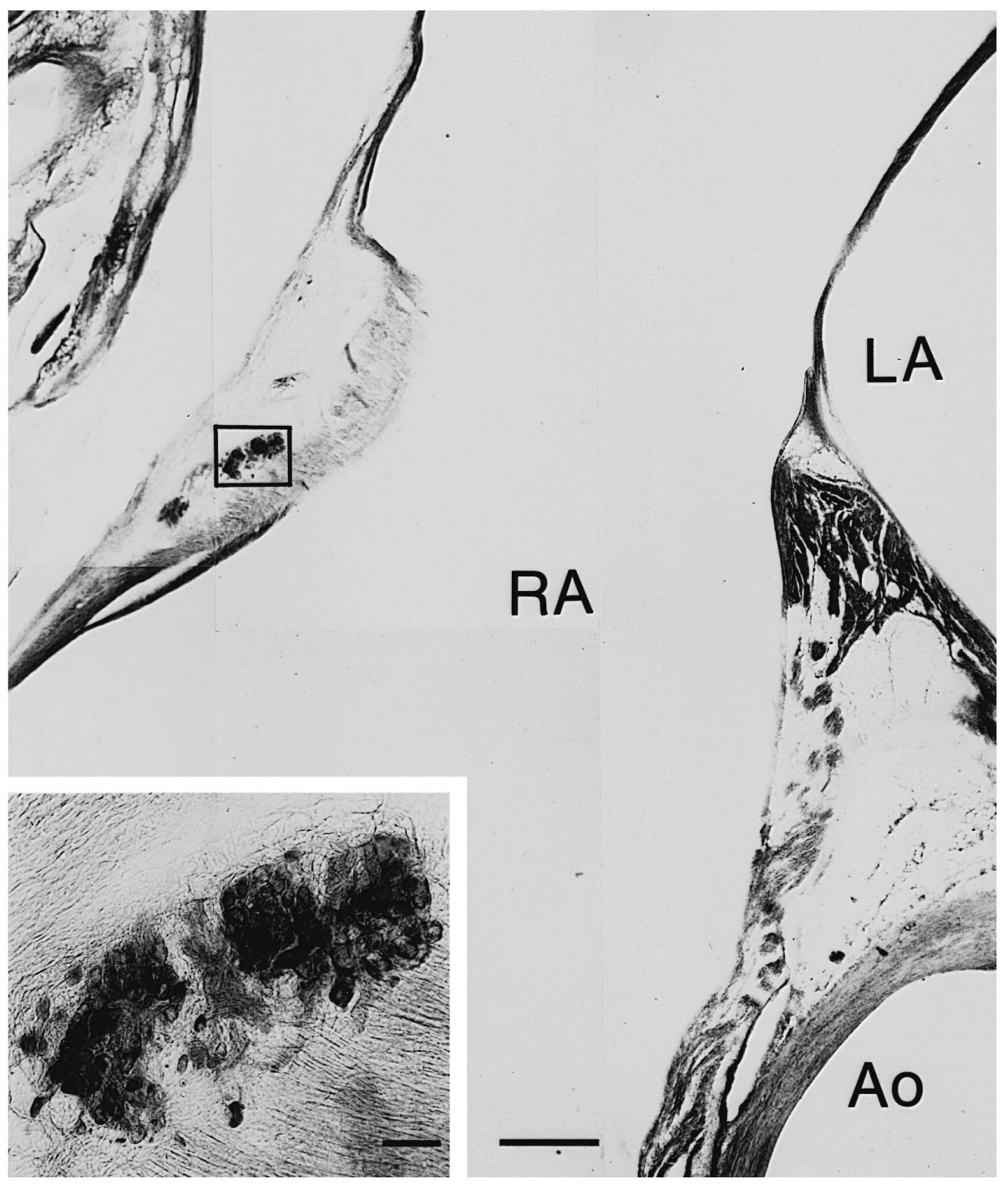

Fig. 3. Photomicrographs of intracardiac ganglionic neurons in the heart, stained with acetylcholinesterase histochemistry. Transverse section of the heart at the level corresponds to Fig. 1, C in a different cat. Inset shows the enlarged view of neuron clusters in the connective tissue of the right atrium (RA). The labelled cells were many and densely stained. Ao, aorta; LA, left atrium. Scale bar in inset $=100 \mu \mathrm{m}$; scale bar $=1 \mathrm{~mm}$. 
HRP (Boehringer) in physiological saline was injected directly into the base between the pulmonary artery and aorta, and the dorsum of the interatrial septum, where vagal postganglionic neurons are located (Calaresu and Louis, 1967). The total injection for each animal was approximately $10 \mu \mathrm{l}$.

\subsubsection{Injection of HRP into the cardiopulmonary branch}

In six cats the lateral incision was made along the fourth rib of the thorax. The cardiopulmonary branch of the vagus nerve near/adjacent to the azygous vein (the right side) or the heart (the left side) was identified as caudal vagal nerves (Phillips et al., 1986). Criteria for a cardiac branch of the vagus nerve included: (i) a branch of the vagus distal to the recurrent laryngeal nerve; (ii) the branch terminated in the cardiopulmonary plexus near the atrium; (iii) electrical stimulation of the nerve produced pronounced bradycardia with a current less than $0.2 \mathrm{~mA}$. In three cats the left cardiopulmonary branch, and in the other three the right branch, were exposed and injected. For each of the cardiopulmonary branches less than $3 \mu \mathrm{l}$ HRP was injected.

\subsubsection{Injection of HRP into the five vagal branches in the thorax}

In 20 cats, a lateral incision was made along the eighth rib of the thorax to expose the two vagus nerves and their four branches (Fig. 1). The right thoracic vagus nerve courses near the heart on the dorsal surface of the esophagus to the left side where right and left branches are formed. The left vagus in the thorax near the heart traverses across the ventral surface of the esophagus to the right side where it splits also into right and left branches. The right branch of the right vagus nerve joins the right branch of the left vagus nerve to form the anterior vagus nerve about $3 \mathrm{~cm}$ from the diaphragm. The left branch of the right vagus nerve joins the left branch of the left vagus nerve to form the posterior vagus nerve about $1 \mathrm{~cm}$ from the diaphragm. The anterior and the posterior vagus nerves descend through the diaphragm to become the anterior and the posterior vagus branches of the abdominal vagus, respectively (Prechtl and Powley, 1985). Approximately $10 \mu 1$ HRP (20\%) were injected directly into the thoracic anterior vagus nerve and each of the four vagal branches; one branch for each cat. No injection was made in the posterior vagus nerve because of technical difficulty in approach.

Injection of HRP to the nerves was made under a stereomicroscope by puncturing the nerves directly with the sharp tip of the Hamilton syringe when the nerves were large. If the nerves were small (e.g. the cardiac pulmonary nerves) injections were made through a glass micropipette with a tip about $30 \mu \mathrm{m}$ in diameter. The rest of the micropipette, which had been sealed with silicone rubber, was attached to Hamilton syringe. The tracer was injected deeply into the nerve at slow rate (30 s). Any leakage, if occurred, was immediately wiped away and washed with saline.

\subsubsection{Injection of HRP into the cervical vagus}

In three cats, the left cervical vagus was exposed and injected with 10-20 $\mu$ l of HRP (20\%) through a Hamilton syringe.

\subsubsection{Demonstration of HRP neurons}

After HRP injection, the chest wall was closed and the animal was allowed to recover from anesthesia and given postoperative care. Four to five days later, the cat was re-anesthetized and perfused transcardially with $0.9 \%$ saline, followed by 21 of a fixative solution containing $2.5 \%$ glutaraldehyde and $1 \%$ paraformaldehyde in $0.1 \mathrm{M}$ phosphate buffer ( $\mathrm{pH} 7.3$ ), and finally washed with 21 of $0.1 \mathrm{M}$ phosphate buffer $(\mathrm{pH} 7.3)$ containing $30 \%$ sucrose at $4^{\circ} \mathrm{C}$. The brain stem was removed and stored in $30 \%$ sucrose solution at $4^{\circ} \mathrm{C}$ for $24 \mathrm{~h}$. Subsequently, the brain stem was serially sectioned at $40 \mu \mathrm{m}$ on a cryostat (Reichert-Jung, 2800 Frigocut E). The sectioned tissues were treated with tetramethylbenzidine (TMB) as the chromogen for the demonstration of HRP reaction product (Mesulam, 1978). The sections were counterstained with

Table 1

The distribution of HRP labelled neurons in the medulla of cats at various levels, rostral (A) or caudal (P) to the obex (in $\mathrm{mm}$ ) after injection of HRP into the subepicardium of the heart or cardiac nerves

\begin{tabular}{|c|c|c|c|c|c|c|c|c|c|c|c|}
\hline Levels & & $\mathrm{P} 0.5$ & A0.0 & A0.5 & A1.0 & A1.5 & A2.0 & A2.5 & A3.0 & A3.5 & $\sum$ \\
\hline \multicolumn{12}{|c|}{ HRP on the subepicardium $(n=6)$} \\
\hline \multirow[t]{2}{*}{ DMV } & $\mathrm{L}$ & 1 & 1 & 3 & 2 & 1 & 2 & 2 & 2 & 0 & 20 \\
\hline & $\mathrm{R}$ & 0 & 1 & 2 & 1 & 1 & 1 & 0 & 0 & 0 & \\
\hline \multirow[t]{2}{*}{$\mathrm{IZ}$} & $\mathrm{L}$ & 0 & 0 & 0 & 0 & 0 & 0 & 0 & 0 & 0 & 2 \\
\hline & $\mathrm{R}$ & 0 & 0 & 1 & 0 & 0 & 1 & 0 & 0 & 0 & \\
\hline \multirow[t]{2}{*}{ NA } & $\mathrm{L}$ & 3 & 19 & 31 & 27 & 18 & 14 & 5 & 2 & 0 & 223 \\
\hline & $\mathrm{R}$ & 1 & 21 & 18 & 19 & 17 & 16 & 9 & 3 & 0 & \\
\hline \multicolumn{2}{|c|}{ Heart total } & 5 & 42 & 55 & 49 & 37 & 34 & 16 & 7 & 0 & $245^{*}$ \\
\hline \multicolumn{12}{|c|}{ HRP on the left cardiac nerve $(n=3)$} \\
\hline DMV & $\mathrm{L}$ & 1 & 0 & 0 & 3 & 0 & 1 & 2 & 1 & 4 & 12 \\
\hline $\mathrm{IZ}$ & $\mathrm{L}$ & 0 & 0 & 0 & 0 & 0 & 0 & 2 & 0 & 0 & 2 \\
\hline \multirow[t]{2}{*}{ NA } & $\mathrm{L}$ & 5 & 4 & 7 & 8 & 19 & 10 & 18 & 8 & 2 & 81 \\
\hline & $\Sigma$ & 6 & 4 & 7 & 11 & 19 & 11 & 22 & 9 & 6 & 95 \\
\hline \multicolumn{12}{|c|}{ HRP on the right cardiac nerve $(n=3)$} \\
\hline DMV & $\mathrm{R}$ & 1 & 4 & 1 & 5 & 1 & 8 & 8 & 6 & 0 & 34 \\
\hline $\mathrm{IZ}$ & $\mathrm{R}$ & 1 & 0 & 3 & 0 & 3 & 1 & 1 & 1 & 1 & 11 \\
\hline \multirow[t]{2}{*}{ NA } & $\mathrm{R}$ & 2 & 6 & 5 & 0 & 4 & 9 & 16 & 2 & 2 & 46 \\
\hline & $\sum$ & 4 & 10 & 9 & 5 & 8 & 18 & 25 & 9 & 3 & 91 \\
\hline $\begin{array}{l}\mathrm{R} \text { and } \mathrm{L} \\
\text { nerve } \\
\text { total }\end{array}$ & & 10 & 14 & 16 & 16 & 27 & 29 & 47 & 18 & 9 & $186^{\mathrm{a}}$ \\
\hline
\end{tabular}

Numbers listed in the table are the total number of labelled neurons counted from three or six animals as indicated.

$n$, Number of animal; DMV, dorsal motor nucleus of vagus; IZ, intermediate zone; NA, nucleus ambiguus. L, left medulla; R, right medulla. $\Sigma$, total number.

${ }^{\mathrm{a}}$ Distribution significantly different $\left(9 \times 2\right.$ contingency table, $\chi^{2}$ test, $P<0.05)$. 
neutral red or thionin and examined under a microscope (Nikon). The labelled cells in every other section were counted under the microscope by marking the $x-y$ position of every neuron on a $x-y$ plotter (Hewlett Packard 7090A) which was coupled to the mechanical stage of the microscope.

\section{Results}

\subsection{Distribution of intracardiac ganglionic neurons}

In three cats, the distributions of intracardiac ganglionic neurons of the heart were determined. The labelled cells were found to be distributed in the connective tissue of large vessels (aorta, pulmonary artery, pulmonary vein, cranial vena cava, caudal vena cava), atrium and atrial septum. The diameter of the neurons varied from 20 to 30 $\mu \mathrm{m}$. Two major groups of neurons were found in the heart. One group was distributed between the aorta, pulmonary artery and right atrium (Fig. 2A-D). The other group was found in the tissue of the atrial septum, the dorsal side of the right atrium near the sinoatrial node (Fig. 2C), or the dorsal atrial septum and two sides of the dorsal atrium (Fig. 2D). Representative photomicrographs of a dense population of neurons on the right side of the right atrium are shown in Fig. 3. No ganglionic cell were found in the ventricle.

\subsection{Distribution of HRP-labelled neurons in medulla}

\subsubsection{Comparison of results between subepicardium and cardiac pulmonary nerve injections}

In six cats, HRP was injected into the subepicardium of the heart between the pulmonary artery and aorta, and the dorsum of the interatrial septum. A total of 245 HRP labelled neurons $(n=6)$ was found bilaterally distributed within three medullary regions, i.e. the NA, the DMV and an intermediate zone (IZ) between the two nuclei. The majority of HRP labelled cells was located in the region within and ventrolateral to the NA $(223 / 245$ or $91.0 \%$, Fig. 9). A few were found in the lateral DMV (20/245 or $8.2 \%)$ and only two in the IZ $(2 / 245$ or $0.8 \%$, Table 1 , Fig. 9). The highest density of the labelled neurons in NA was at the region $0.5 \mathrm{~mm}$ rostral to the obex. In three cats, HRP was injected into the left cardiopulmonary vagus
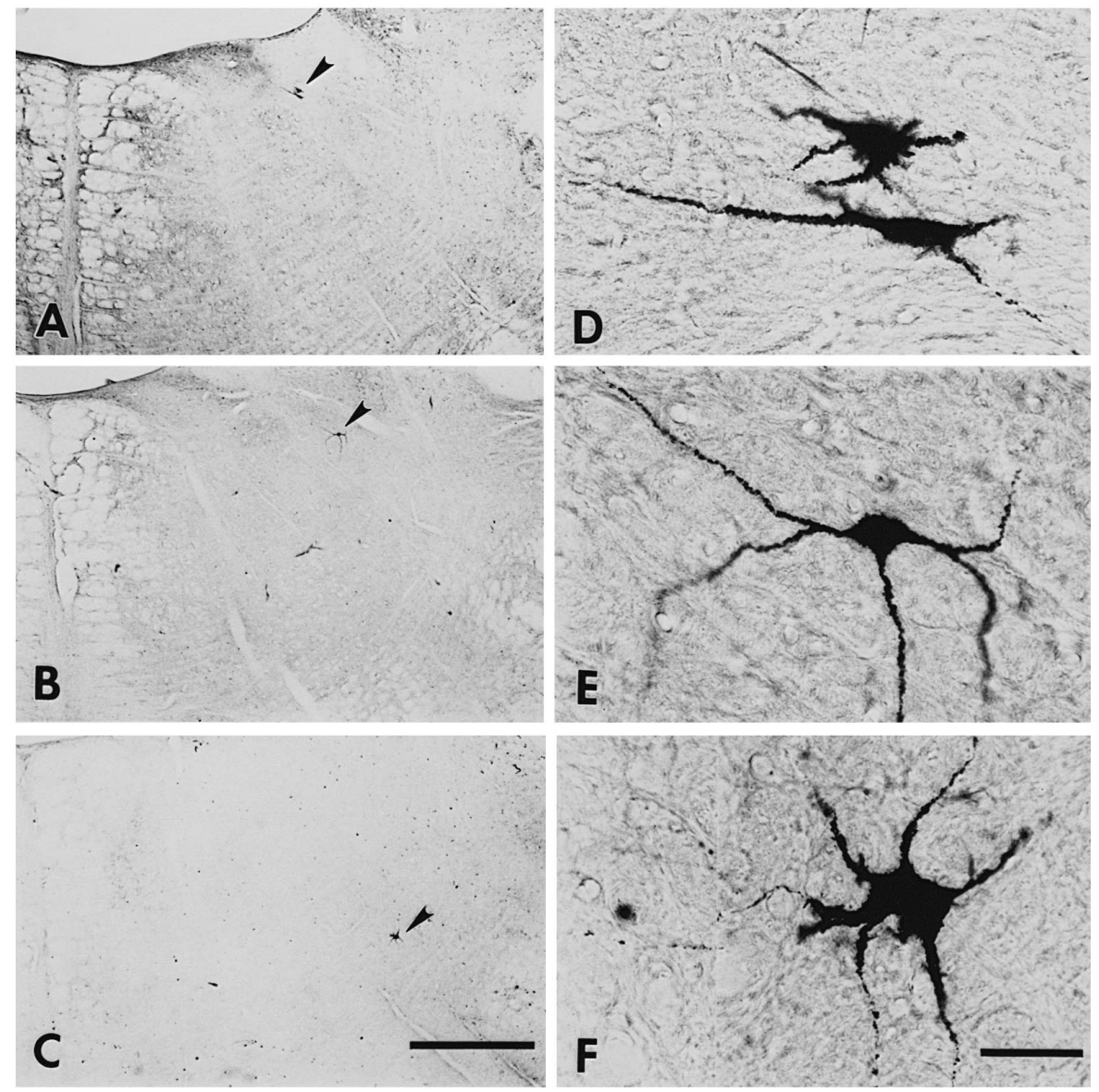

Fig. 4. HRP labelled neurons found in ipsilateral DMV (A), intermediate zone (B) and NA (C) after injection of HRP into the right cardiac branch of vagus nerve. D-F show the enlarged HRP labelled cells found in DMV, IZ and NA, respectively. Scale bar in C, $1 \mathrm{~mm}$, applies to A-C. Scale bar in F, $50 \mu \mathrm{m}$, applies to D-F. 

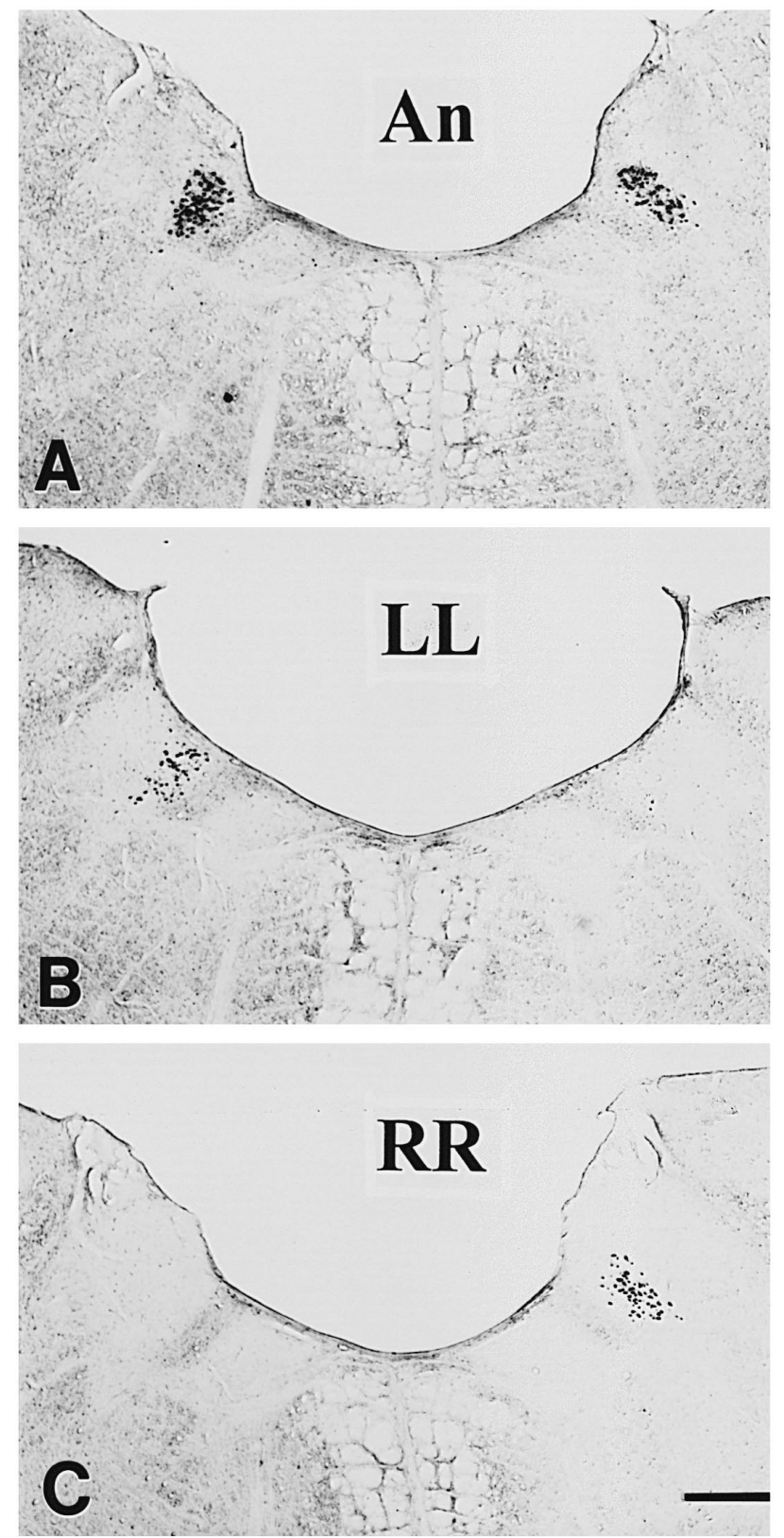

Fig. 5. HRP labelled neurons were found in DMV bilaterally after injection into the anterior vagus nerve (An). They were found ipsilateral to the injection side when HRP was injected into either the left branch of the left thoracic vagus (LL) or the right branch of the right thoracic vagus (RR). Scale bar $=500 \mu \mathrm{m}$.

branch. After the injection, labelled neurons were found ipsilaterally in the left NA (Fig. 4C,F), DMV (Fig. 4A and D) and IZ (Fig. 4B and E) but predominantly in the NA. In another three cats receiving HRP injection into the right cardiopulmonary vagus branch, labelled neurons were found ipsilaterally in the right NA, DMV and IZ, also with predominance in the NA (Table 1). When these two nerve injected groups were combined, it was found that labelled neurons were significantly higher in NA $(127 / 186$ or $68.3 \%)$ than in DMV (46/186 or $24.7 \%)$. The density of HRP labelled neurons in the nerve injected group reached a maximum in the medulla at $2.5 \mathrm{~mm}$ rostral to the obex. In contrast, the density of labelled neurons in the heart injected group were at a maximum at the level $0.5 \mathrm{~mm}$ rostral to the obex. The distribution of the labelled neurons between the nerve and the heart injected groups is significantly different ( $\chi^{2}$ test, $P<0.05$, Table 1$)$.

\subsubsection{Injection of HRP into the five vagal branches in the lower thorax}

In a group of 20 cats, HRP was injected into each of the five vagal branches in the lower thorax, namely, the anterior vagus, the right and left branches of the right thoracic vagus and the right and left branches of the left thoracic vagus $(n=4$ each). In all these injections, HRP labelled cells were consistently and exclusively localized within the DMV and other region of the brain stem (Figs. 6 and 9$)$.

Figs. 5 and 6 show representative photomicrographs of HRP labelled neurons on both sides of DMV after injection of HRP into the lower thoracic vagus nerve. The number of labelled cells is large (Figs. 7 and 8). When HRP was injected into either the right or the left branches

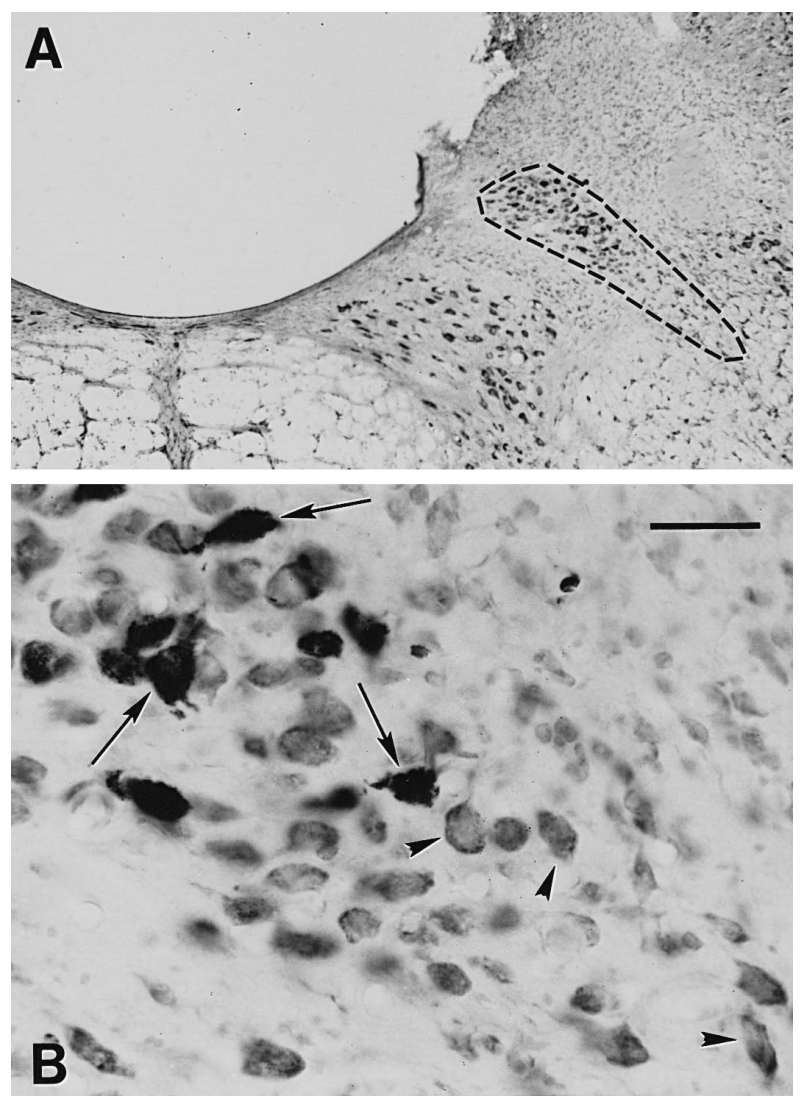

Fig. 6. A representative example showing the presence of that HRP labelled neurons in the medial DMV after injection in the lower thoracic vagus nerve. In $\mathrm{A}$, it shows a lower while in $\mathrm{B}$, a higher magnification of labelled neurons in the right DMV, when HRP was injected into the anterior vagus nerve. The boundary of the DMV is marked with a dotted line in A. Note the large number of HRP labelled neurons (arrows) located medially, while no neurons labelled in the lateral DMV (arrowheads). Scale bar in $\mathrm{A}=160 \mu \mathrm{m}$ and in $\mathrm{B}=40 \mu \mathrm{m}$. 


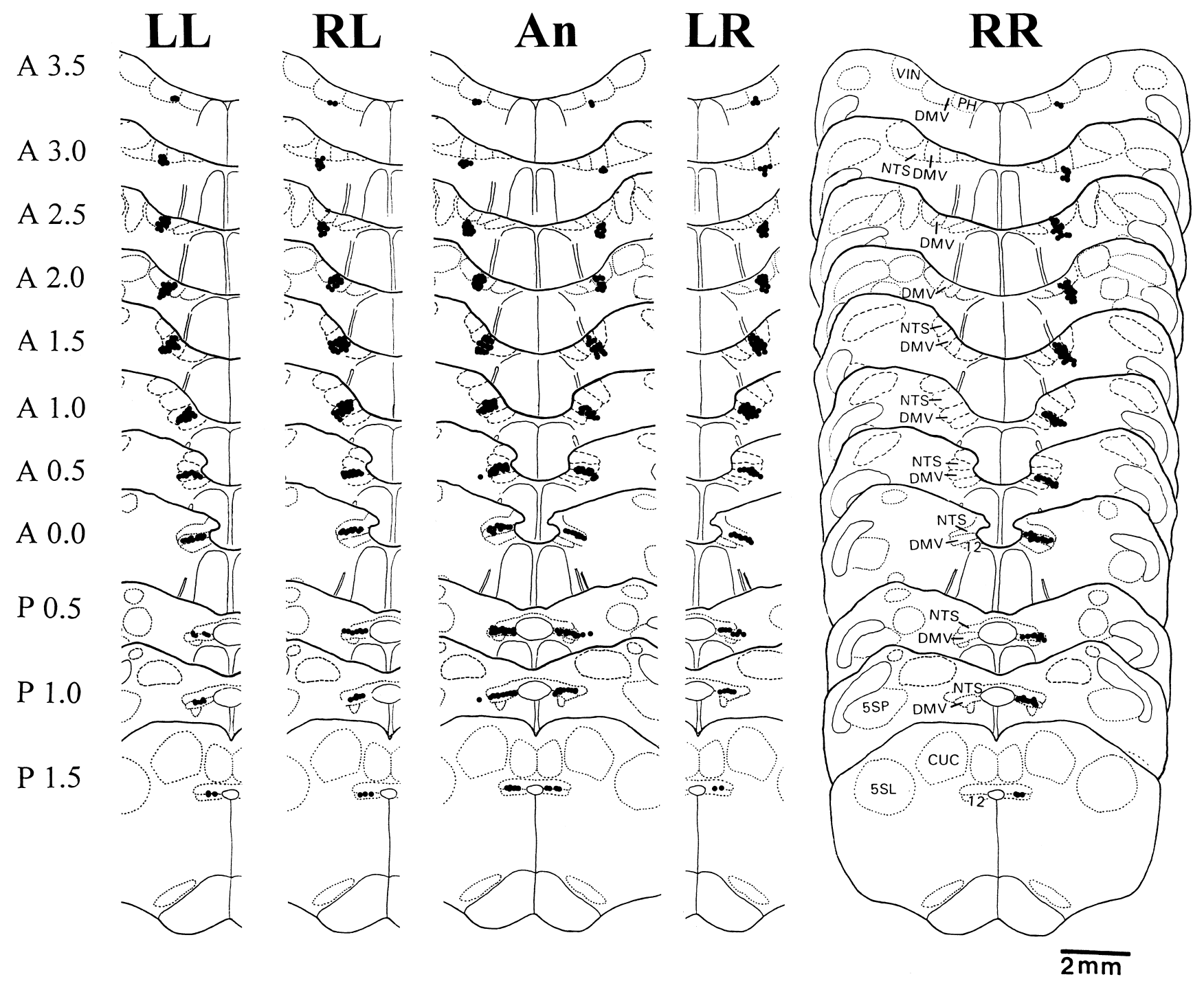

Fig. 7. Composite drawing showing the distribution of HRP labelled neurons in DMV throughout various levels of the medulla from $3.5 \mathrm{~mm}$ rostral to the obex (A3.5) to $1.5 \mathrm{~mm}$ caudal to the obex (P1.5) after injection of HRP in one animal, each in one of the five vagal branches. After injection of HRP into the left (LL) and right (RL) branches of the left thoracic vagus, all labelled neurons were found in the left DMV. After injection of HRP into the anterior vagus (An), HRP labelled cells were found in DMV on both sides. After HRP was injected into the right (RR) and left (LR) branches of the right vagus, all HRP-labelled cells were observed in the right DMV. A, rostral; P, caudal to the obex.

of the left vagus in the thorax, HRP-labelled cells were observed exclusively within the left DMV (Fig. 5LL, Fig. $7 \mathrm{LL}, \mathrm{RL})$. When HRP was injected into either the right or the left branch of the right vagus in the thorax, labelled neurons were found exclusively in the right DMV (Fig. 5RR, Fig. 7LR and RR). Labelled cells were distributed in DMV along the rostro-caudal axis throughout an extent of about $5 \mathrm{~mm}$, from $1.5 \mathrm{~mm}$ caudal to and $3.5 \mathrm{~mm}$ rostral to the obex. They were most abundant at the level 1-1.5 mm rostral to the obex. The majority of HRP labelled cells were found in the medial part of DMV (Figs. 6 and 9).

\subsubsection{Injection of HRP into the cervical vagus nerve}

In three cats HRP was injected into the left cervical vagus nerve. A total of 8175 HRP labelled cells were found. They were distributed ipsilaterally in three medullary regions, i.e. $74 \%(6078 / 8175)$ in the DMV, $23 \%(1839 / 8175)$ in NA and $3 \%(252 / 8175)$ in IZ (Fig. 9).

\section{Discussion}

The most important findings of the present study are as follows: 1. Elucidation of the origin and the course of the vagus nerve in the lower thorax of cats; 2 . Demonstration of differential patterns of retrogradely labelled cells in the medulla following HRP injection into the heart and the cardiopulmonary nerve; 3 . Demonstration of the primary location of the cardiac vagal preganglionic neurons in NA at the obex level by the use of a more specific procedure of intracardial injection, and of the location of the GI vagal 

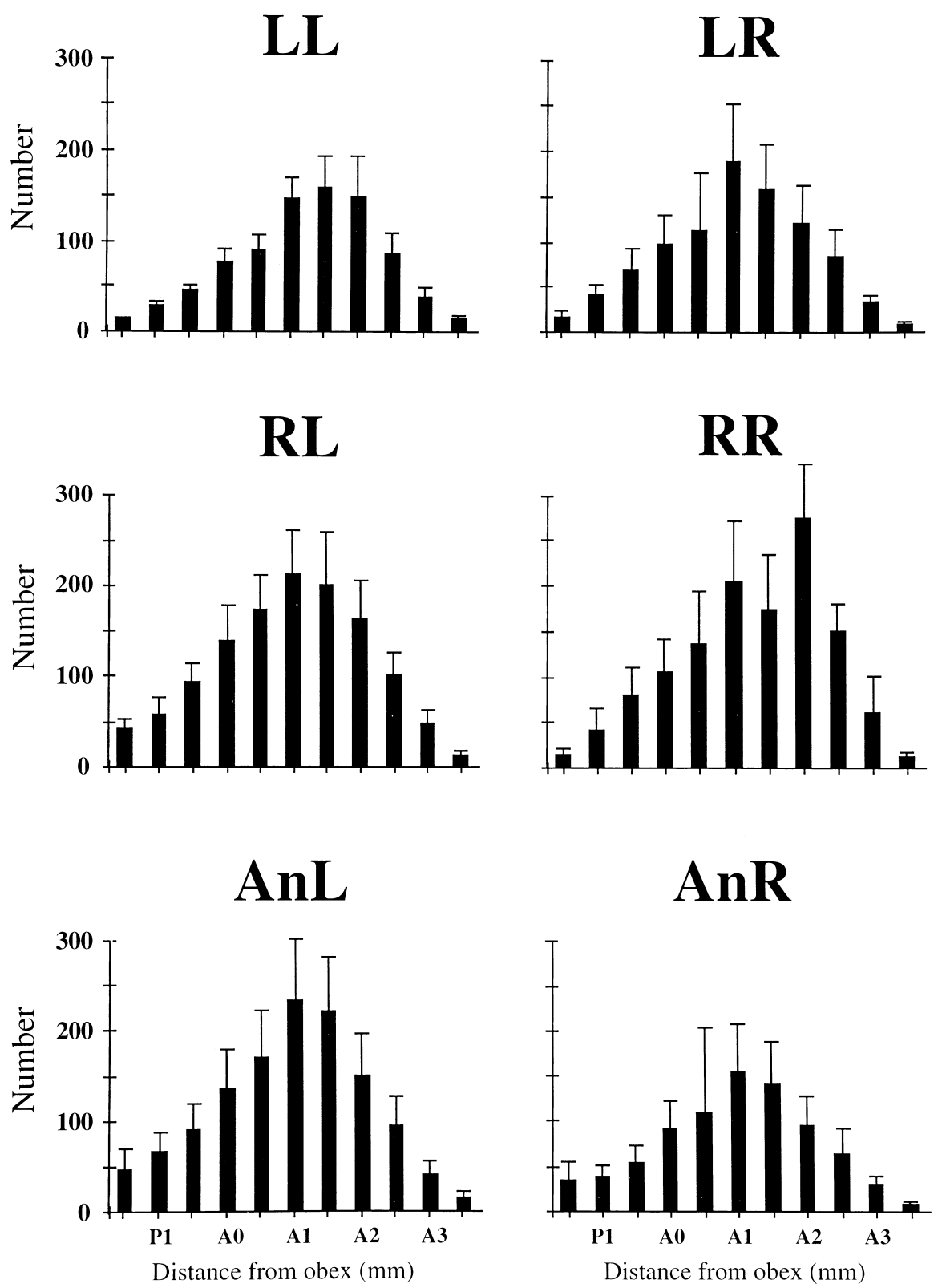

Fig. 8. Distribution of HRP labelled neurons in the dorsal motor nucleus of vagus at the rostrocaudal axis, rostral (A) or caudal (P) to the obex (in mm) after injection of the tracer into the lower thoracic vagal branches. Numbers listed in the histogram are the average number of labelled neurons \pm SE counted from four animals for each nerve in a total of 20 cats. AnL, labelled neurons in left medulla after anterior vagus nerve injection; AnR, labelled neurons in right medulla after anterior vagus nerve injection; RL, right branch of the left thoracic vagus; LL, left branch of the left thoracic vagus; LR, left branch of the right thoracic vagus; RR, right branch of the right thoracic vagus.

neurons in the medial DMV at a level 1-2 $\mathrm{mm}$ rostral to the obex.

\subsection{Origin of GI vagal preganglionic neuron}

The NA consists of two components, i.e. the ventral motor nuclei of the glossopharyngeal nerve and the nuclei of the vagus nerve (Kalia and Mesulam, 1980). Besides cardiovascular functions, this nucleus has been considered to be involved in other functions including swallowing, vocalization, and respiration. NA has been thought to play a role in the integration of GI functions. For example, Kalia and Mesulam (1980) injected HRP into the cardia of the stomach in cats and observed labelled cells in NA. Takayama et al. (1982) reported that injection of HRP into the cardia of stomach in rats produced bilaterally labelled cells scattering in NA. Yamamoto et al. (1977) observed that after HRP injection into the pyloric ring in cats some cells were labelled in the medial NTS. On the other hand, many investigators (Dennison et al., 1981; Kalia and Mesulam, 1980; Okumura and Namiki, 1990; Pagani et al., 1984; Shapiro and Miselis, 1985; Stuesse, 1982) have observed intense labelling of cells in DMV without la- 

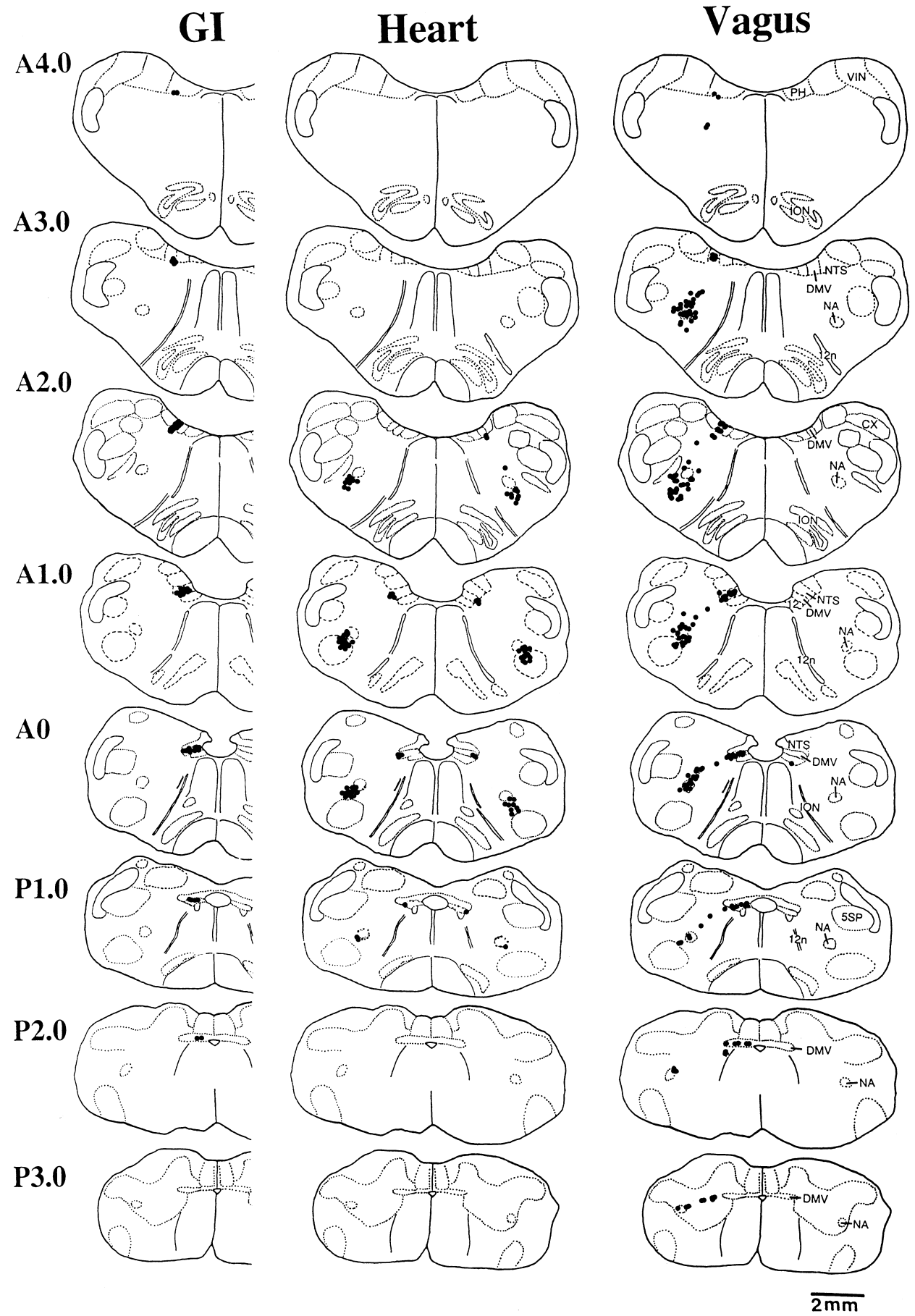

Fig. 9. Summary diagram of the topographic organization of GI vs. heart preganglionic vagal neurons. A representative result from one animal was plotted for each column. An animal injected in the left branch of the left thoracic vagus was used to represent the GI group. Each dot represents one HRP labelled neuron. Note the strict laterality in neurons project to the cervical vagus (vagus) and the lower thoracic vagus (GI).

belling in either NA or NTS after injection of retrograde tracers into the stomach. Norman et al. (1985) reported that in cats labelled cells were observed in DMV after HRP injection into the GI tract, at the level of $1.8 \mathrm{~mm}$ rostral to $1.0 \mathrm{~mm}$ caudal to the obex, with the most intense labelling in the region $0.7-1.0 \mathrm{~mm}$ rostral to the obex. Pagani et al. $(1985,1988)$ reported that in cats after HRP injection into the stomach labelled neurons occupy a length of $5 \mathrm{~mm}$ in the DMV, with a highest density of labelling in the area from the obex to $2.0 \mathrm{~mm}$ rostral to the obex.

After injection of HRP into the duodenum of cats (Kalia and Mesulam, 1980; Todo et al., 1977) and rats (Shapiro and Miselis, 1985) the origin of vagal preganglionic neurons innervating GI tract has been found bilaterally in DMV. Several papers (Berthoud et al., 1991; Kalia and Mesulam, 1980; Satomi et al., 1978) reported a similar 
result after injection of HRP into the jejunum and ileum in cats. Injection of HRP into the proximal and the midportion of the colon of the cat results in labelling of cells solely in the DMV (Satomi et al., 1978). Injection of HRP into the descending colon of the cat results in labelling of cells in the DMV and in the intermediolateral cell column of the $\mathrm{S}_{2-3}$ level (Satomi et al., 1978). However, no labelled cells were observed in the DMV after HRP injection into the distal colon.

In the present study, HRP was selectively injected into the five branches of the vagus nerves at the level of lower thorax. Efferent vagal fibers at this level innervate the lower esophagus and all the abdominal vagal targets. Our results showing confinement of labelled neurons to the DMV are in line with most of studies cited above. Thus, a conclusion can be drawn that the vagal preganglionic fibers to abdominal organs of the cat take their origin from DMV. A further conclusion is that the left and right vagus nerves begin to mix only at the lower thoracic level. Strict laterality occurs rostral to this level. Caudal to this level, visceral targets were innervated by vagal nerves originating from both sides of the DMV.

The concept of a possible viscerotropic organization of DMV neurons innervating the abdominal viscera still remains controversial. The study of Yamamoto et al. (1977) in cats demonstrated that the stomach receives vagal fibers from neurons in DMV and that these fibers are organized bilaterally and longitudinally along the neuraxis of the nucleus mainly in its medial portion. McConnie et al. (1988) reported that after application of cholera toxin conjugated HRP to the cardia, the body or the pylorus of the stomach in rats, retrograde labelling cells can be found in the medial two thirds of DMV. Other studies including those in fish (Withington-Wray et al., 1986), rat (Altschuler et al., 1991; Nosaka et al., 1982; Yoshida et al., 1989), and cat (Pagani et al., 1985, 1988; Satomi et al., 1978) have demonstrated that the cells in DMV are organized into specific sites, innervating different parts of the abdominal viscera. This suggests a possible evidence of a viscerotropic organization of neurons in the DMV of these animals. Some authors maintain that vagal targets do not show discrete or topographic representation, at least in the DMV complex of the rat (Shapiro and Miselis, 1985), and the cat (Hamlin and Smith, 1968; Kalia and Mesulam, 1980). The present results confirm that neurons in DMV that innervate the GI tract and the cardiopulmonary structures are topographically organized. A larger portion of neurons that innervate the GI tract are located in the medial portion of the DMV, while a smaller portion of neurons that innervate the lungs and the heart are located in the lateral edge of the DMV.

\subsection{Origin of cardiac vagal preganglionic neuron}

Numerous studies have attempted to identify the origin of parasympathetic preganglionic neurons innervating the heart. Previous studies have utilized injections of retro- grade tracers into the cardiopulmonary nerve (Hopkins and Armour, 1982; Maqbool et al., 1991; Sugimoto et al., 1979), myocardium (Izzo et al., 1993), atria or ventricles (Standish et al., 1994; Stuesse, 1982) or intracardiac ganglia (Massari et al., 1995). Controversy, however, still remains regarding what proportion of the NA and DMV contributes to the preganglionic neuronal pool of the cardiac vagus. A possible source of variation may arise from different methods of injection, particularly when injections are made at different target sites. Thus, we compared the results derived from injections in the cardiopulmonary nerve and epicardium. Prior to HRP application, the injection sites in epicardium were determined first by localization of the intracardiac ganglia by means of histochemical staining technique for acetylcholinesterase. The distribution of the intracardiac ganglionic neurons found in the present study is consistent with the findings of Calaresu and Louis (1967) and Yuan et al. (1994).

The cardiopulmonary nerves contain many cardiac and pulmonary fibers (McAllen and Spyer, 1978). Injection into these nerves, therefore, labelled both cardiac and pulmonary vagal preganglionic neurons. On the other hand, epicardiac injection was more specific, and labelled only cardiac vagal neurons. The difference in the distribution of medullary neurons is consistent with the results of McAllen and Spyer (1976), Hopkins and Armour (1982), Maqbool et al. (1991) and Cabot et al. (1991). They all found a rostrocaudal organization of pulmonary vs. cardiac vagal preganglionic neurons in the NA. Each NA of the cat is approximately 6-7 $\mathrm{mm}$ long. The pulmonary neurons are located more rostrally and the cardiac neurons caudally. In addition, our results confirm the previously described division of the nucleus into a rostral compact formation and a caudal diffuse formation occupying the remainder of the nucleus, with the cardiac vagal neurons residing in the ventrolateral part of the caudal NA (Kitamura et al., 1993; Lawn, 1966).

Another interesting finding of the present study concerns the relative importance of the NA vs. DMV in the vagal innervation of the heart. In cardiopulmonary nerve injected cats, we found a significantly higher portion of DMV neurons labelled than that of the subepicardium injected cats $(24.7 \%$ vs. $8.2 \%)$. This suggests that either the lateral DMV contains more pulmonary vagal preganglionic neurons or that nerve injection is a more effective means in labelling DMV neurons. Since nerve injection has not been shown to preferentially label small neurons in the central nervous system, we consider the first possibility is a more likely explanation. Further supporting evidence for this explanation comes from our finding that following injection of HRP into the cardiopulmonary nerve the highest density of HRP labelled neurons was in the rostral medulla (at the level 2-2.5 $\mathrm{mm}$ rostral to the obex), whereas the HRP labelled neurons following injection in the subepicardium region were concentrated at a more caudal level (0-1 $\mathrm{mm}$ rostral to the obex). 
A quantitative comparison of the relative contribution of the DMV vs. NA in the cardiac vagal neuron pool was made possible by the cervical vagus injected animals. An average of 2725 neurons per animal was labelled by cervical vagal injection, 2028 of these were in the DMV and 613 in the NA. This was the total population to thoracic and abdominal organs. An injection into the heart produced an average of 40.8 labelled cells in the medulla per animal, 37.2 of these were in the NA and only 3 were in the DMV, representing $0.15 \%$ of the total DMV cells. In the densest zone of the cardiac vagal neurons, i.e. within the level 0-1 mm rostral to obex, we found an average of 22.7 NA cells and 1.7 DMV cells that projected to the heart. These were $8.3 \%$ and $0.15 \%$ of the vagal NA and DMV cells population (273 and 1154) respectively. Although cardiac injection may only label a small portion of the total cardiac vagal preganglionic neurons because the widely dispersed nature of the intracardiac ganglionic neurons, the difference in proportion (55 times more labelled cells in the NA vs. DMV) should be accurate. Quantitatively, only three DMV cells per animal were found projecting to the heart. This is in striking contrast to the average 1579 labelled cells in the DMV per animal after injection of the same tracer into the lower thoracic branches. Our conclusions, therefore, are that most DMV vagal neurons project to the GI, only a very small portion of the lateral DMV neurons project to the heart, and that NA devotes more than 50 times of its neurons to the heart than the DMV.

In summary, findings of the present experiment show that in cats the left and right vagus nerves begin to mix at the lower thoracic level with a very simple pattern, i.e. they each bifurcate, the inner branch of each nerve crosses sides to join the lateral branch of the other nerve, so that vagal branches caudal to the diaphragm has mixed origins from both sides of the medulla. Whether the bifurcation pattern has functional significance awaits further study. The second conclusion from the present experiment is that preganglionic neurons of the thoracic vagus are organized topographically in medulla oblongata. Neurons innervating the heart are located primarily in the NA, while those innervating the GI tract are located in the medial DMV. This will help clinical diagnosis in correlating the cardiovascular or GI symptoms with the involvement of lesion in the region of DMV or NA.

\section{Acknowledgements}

The authors thank Dr S.D. Wang for helpful advice in preparation of the illustrations and J.J. Pan for preparation of the manuscript. Thanks are also due to Drs S.H. Ngai, Professor Emeritus of the College of Physicians and Surgeons, Columbia University, USA and C.V. Weaver, Professor of $\mathrm{Fu}$ Jen Catholic University for their helpful comments on the manuscript. This study was supported in part by the Institute of Biomedical Sciences, Academia Sinica, Foundation of Biomedical Sciences, Shih-Chun Wang Research Fund, and National Sciences Council, Republic of China Grants; NSC83-0412-B-033-003 and NSC84-2331-B-033-002.

\section{References}

Altschuler, S.M., Ferenci, D.A., Lynn, R.B., Miselis, R.R., 1991. Representation of the cecum in the lateral dorsal motor nucleus of the vagus nerve and commissural subnucleus of the nucleus tractus solitarii in rat. J. Comp. Neurol. 304, 261-274.

Berthoud, H.R., Carlson, N.R., Powley, T.L., 1991. Topography of efferent vagal innervation of the rat gastrointestinal tract. Am. J. Physiol. 260, R200-R207.

Bieger, D., Hopkins, D.A., 1987. Viscerotropic representation of the upper alimentary tract in the medulla oblongata in the rat: the nucleus ambiguus. J. Comp. Neurol. 262, 546-562.

Cabot, J.B., Carroll, J., Bogan, N., 1991. Localization of cardiac parasympathetic preganglionic neurons in the medulla oblongata of pigeon, Columba livia: a study using fragment $\mathrm{C}$ of tetanus toxin. Brain Res. 544, 162-168.

Calaresu, F.R., Louis, A.J.S., 1967. Topography and numerical distribution of intracardiac ganglion cells in the cat. J. Comp. Neurol. 131, 55-66.

Ciriello, J., Calaresu, F.R., 1982. Medullary origin of vagal preganglionic axons to the heart of the cat. J. Auton. Nerv. Syst. 5, 9-22.

Cohen, D.H., Schnall, A.M., Macdonald, R.L., Pitts, L.H., 1970. Medullary cells of origin of vagal cardioinhibitory fibers in the pigeon: I. Anatomical studies of peripheral vagus nerve and the dorsal motor nucleus. J. Comp. Neurol. 140, 299-320.

Dennison, S.J., O’Connor, B.L, Aprison, M.H., Merritt, V.E., Felten, D.L., 1981. Viscerotropic localization of preganglionic parasympathetic cell bodies of origin of the anterior and posterior subdiaphragmatic vagus nerves. J. Comp. Neurol. 197, 259-269.

Ewart, W.R., Jones, M.V., King, B.F., 1988. Central origin of vagal nerve fibers innervating the fundus and corpus of the stomach in rat. J. Auton. Nerv. Syst. 22, 115-128.

Fox, E.A., Powley, T.L., 1985. Longitudinal columnar organization within the dorsal motor nucleus represents separate branches of the abdominal vagus. Brain Res. 341, 269-282.

Geis, G.S., Wurster, R.D., 1980. Cardiac responses during stimulation of the dorsal motor nucleus and nucleus ambiguus in the cat. Circ. Res. 46, 606-611.

Getz, B., Sirnes, T., 1949. The localization within the dorsal motor vagus nucleus: an experimental investigation. J. Comp. Neurol. 90, 95-110.

Gwyn, D.G., Leslie, R.A., Hopkins, D.A., 1985. Observations on the afferent and efferent organization of the vagus nerve and the innervation of the stomach in the squirrel monkey. J. Comp. Neurol. 239, $163-175$.

Hamlin, R.L., Smith, C.R., 1968. Effects of vagal stimulation on S-A and A-V nodes. Am. J. Physiol. 215, 560-568.

Hoff, H.E., 1936. Vagal stimulation before the Webers. Ann. Med. Hist. $8,138$.

Hopkins, D.A., 1987. The dorsal motor nucleus of the vagus nerve and the nucleus ambiguus: structure and connections. In: Hainsworth, R., McWilliams, P.N., Mary, D.A.S.G. (Eds.), Cardiogenic Reflexes. Oxford Univ. Press, Oxford, pp. 185-203.

Hopkins, D.A., Armour, J.A., 1982. Medullary cells of origin of physiologically identified cardiac nerves in the dog. Brain Res. Bull. 8, 359-365.

Hopkins, D.A., Ellenberger, H.H., 1994. Cardiorespiratory neurons in the medulla oblongata: input and output relationships. In: Armour, J.A., Ardell, J.L. (Eds.), Neurocardiology. Oxford Univ. Press, New York, pp. 277-307. 
Izzo, P.N., Deuchars, J., Spyer, K.M., 1993. Localization of cardiac vagal preganglionic motoneurones in the rat: immunocytochemical evidence of synaptic inputs containing 5-hydroxytryptamine. J. Comp. Neurol. 327, 572-583.

Jordan, D., Khalid, M.E.M., Schneiderman, N., Spyer, K.M., 1982. The location and properties of preganglionic vagal cardiomotor neurons in the rabbit. Pflug. Arch. 395, 244-250.

Jou, M.J., Wen, C.Y., Shieh, J.Y., 1993. Localization of the stomach-projecting neurons in the dorsal motor nucleus of the vagus nerve in the guinea pig. J. Auton. Nerv. Syst. 43, 201-206.

Kalia, M., Mesulam, M.M., 1980. Brain stem projections of sensory and motor components of the vagus complex in the cat: I. The cervical vagus and nodose ganglia. J. Comp. Neurol. 193, 435-465.

Kalia, M., Sullivan, J.M., 1982. Brain stem projection of sensory and motor components of the vagus nerve in the rat. J. Comp. Neurol. 211, 248-264.

Karim, M.A., Shaikh, E., Ismail, T.J., 1984. The organization of the gastric efferent projections in the brainstem of monkey: an HRP study. Brain Res. 293, 231-240.

Kitamura, S., Nagase, Y., Chen, K., Shigenaga, Y., 1993. Nucleus ambiguus of the rabbit: cytoarchitectural subdivision and myotopical and neurotopic representations. Anat. Rec. 237, 109-123.

Laughton, W.B., Powley, T.L., 1987. Localization of efferent function in the dorsal motor nucleus of the vagus. Am. J. Physiol. 252, R15-R25.

Lawn, A.M., 1966. The nucleus ambiguus of the rabbit. J. Comp. Neurol. 127, 307-320.

Maqbool, A., Batten, T.F., William, P.N., 1991. Motoneurons and vagal afferents in the cat: a combined HRP tracing and immunogold labeling study. Eur. J. Neurosci. 3, 501-513.

Massari, V.J., Johnson, T.A., Gatti, P.J., 1995. Cardiotopic organization of the nucleus ambiguus? An anatomical and physiological analysis of neurons regulations regulating atrioventricular conduction. Brain Res. 679, 227-240.

McAllen, R.M., Spyer, K.M., 1976. The location of cardiac vagal preganglionic motoneurones in the medulla of cat. J. Physiol. (London) 258 187-204.

McAllen, R.M., Spyer, K.M., 1978. Two types of vagal preganglionic motoneurones projecting to the heart and lungs. J. Physiol. (London) 282, 353-364.

McConnie, R.M., Bao, X., Altschuler, S.M., Miselis, R.R., 1988. Visceral topography in the dorsal motor nucleus (DMN): brain stem motor projections from the rat stomach. Gastroenterology 94, A294.

Mesulam, M.M., 1978. Tetramethyl-benzidine for horseradish peroxidase neurochemistry: a non-carcinogenic blue reaction-product with superior sensitivity for visualizing neural afferents and efferents. J. Histochem. Cytochem. 26, 106-117.

Norman, W.P., Pagani, F.D., Ormesee, H.S. III, Kasbekar, D.K., Gillis, R.A., 1985. Use of horseradish peroxidase to identify hindbrain sites which influence gastric motility in the cat. Gastroenterology 88 , 701-705

Nosaka, S., Yasumaga, K., Tamai, S., 1982. Vagal cardiac preganglionic neurons: distribution, cell types, and reflex discharges. Am. J. Physiol. 243, R92-R98.

Okumura, T., Namiki, M., 1990. Vagal motor neurons innervating the stomach are site-specifically organized in the dorsal motor nucleus of the vagus nerve in rats. J. Auton. Nerv. Syst. 29, 157-162.

Pagani, F.D., Norman, W.P., Kasbekar, D.K., Gillis, R.A., 1984. Effects of stimulation of nucleus ambiguus complex on gastroduodenal function. Am. J. Physiol. 246, G253-G262.
Pagani, F.D., Norman, W.P., Kasbekar, W.P., Gillis, R.A., 1985. Localization of sites within dorsal motor nucleus of vagus that affect gastric motility. Am. J. Physiol. 249, G73-G84.

Pagani, F.D., Norman, W.P., Gillis, R.A., 1988. Medullary parasympathetic projections innervate specific sites in the feline stomach. Gastroenterology 95, 277-288.

Phillips, J.G., Randall, W.C., Armour, J.A., 1986. Functional anatomy of the major cardiac nerves in cats. Anat. Rec. 214, 365-371.

Prechtl, J.C., Powley, T.L., 1985. Organization and distribution of the rat subdiaphragmatic vagus and associated paraganglia. J. Comp. Neurol. 235, 182-195.

Rinaman, L., Miselis, R.R., 1987. The organization of vagal innervation of rat pancreas using cholera toxin-horseradish peroxidase conjugate. J. Auton. Nerv. Syst. 21, 109-125.

Satomi, H., Yamamoto, T., Ise, H., Takatoma, H., 1978. Origins of the parasympathetic preganglionic fibers to the cat intestine as demonstrated by the horseradish peroxidase method. Brain Res. 151, 571578

Shapiro, R.E., Miselis, R.R., 1985. The central organization of the vagus nerve innervating the stomach of the rat. J. Comp. Neurol. 238, 473-488.

Standish, A., Enquist, L.W., Schwaber, J.S., 1994. Innervation of the heart and its central medullary origin defined by viral tracing. Science 263, 232.

Stuesse, S.L., 1982. Origins of cardiac vagal preganglionic fibers: a retrograde transport study. Brain Res. 236, 15-25.

Stuesse, S.L., 1984. Projections to the cardioinhibitory region of the nucleus ambiguus of rat. J. Comp. Neurol. 229, 271-278.

Sugimoto, S., Itoh, K., Mizuno, N., Nomura, S., Konishi, A., 1979. The site of origin of cardiac preganglionic fibers of the vagus nerve: an HRP study in the cat. Neurosci. Lett. 12, 53-58.

Takayama, K., Ishikawa, N., Miura, M., 1982. Site of origin and termination of gastric vagus preganglionic neurons: an HRP study in the rat. J. Auton. Nerv. Syst. 6, 211-223.

Todo, K., Yamamoto, T., Satomi, H., Ise, H., Takatama, H., Takahashi, K., 1977. Origin of vagal preganglionic fibers to the sino-atrial and atrio-ventricular node regions in the cat heart as studied by the horseradish peroxidase method. Brain Res. 130, 545-555.

Williford, D.J., Ormsbee, H.S., Norman, W.P., Harmon, J.W., Gorvey, T.Q., Dimicco, J.A., Gillis, R.A., 1981. Hindbrain GABA receptors influence parasympathetic outflow to the stomach. Science 214, 193194

Withington-Wray, D.J., Roberts, B.L., Taylor, E.W., 1986. The topographical organization of the vagal motor column in the elasmobranch fish Scyliorhinus canicula L. J. Comp. Neurol. 248, 95-104.

Woolf, N.J., Butcher, L.L., 1981. Cholinergic neurons in the caudateputamen complex proper are intrinsically organized: a combined Evans blue and acetylcholinerase analysis. Brain Res. Bull. 7, 487507

Yamamoto, T., Satomi, H., Ise, H., Takahashi, K., 1977. Evidence of the dual innervation of the cat stomach by the vagal dorsal motor and medial solitary nuclei as demonstrated by the horseradish peroxidase method. Brain Res. 122, 125-131.

Yoshida, J., Polley, E.H., Nyhus, L.M., Donahue, P.E., 1989. Brain stem topography of vagus nerve to the greater curvature of the stomach. J. Surg. Res. 46, 60-69.

Yuan, B.X., Ardell, J.L., Hopkins, D.A., Losier, A.M., Armour, J.A., 1994. Gross and microscopic anatomy of the canine intrinsic cardiac nervous system. Anat. Rec. 239, 75-87. 